\title{
Comments on 'Sweep algorithm for solving optimal control problem with multi-point boundary conditions' by M Mutallimov, R Zulfuqarova, and L Amirova
}

Fikret A Aliev

"Correspondence:

f_aliev@yahoo.com Institute of Applied Mathematics,

Baku State University, Baku,

Azerbaijan

\begin{abstract}
A counter example is given for the solution of the linear-quadratic optimization problem with three-point boundary conditions. The example shows that the solution obtained in (Mutallimov et al. in Adv. Differ. Equ. 2015:233, 2015) by using a sweep method is not optimal.
\end{abstract}

Keywords: sweep algorithm; optimization; three-point boundary conditions

\section{Introduction}

In [1] the linear-quadratic optimization problem with multi-point boundary conditions, both in the continuous and the discrete cases, are considered. The sweep method [2, 3], which generalizes the results [4] for the two-point boundary conditions is given in [5]. However, the results obtained for the discrete case [1] are not optimal.

Not passing to the illustration of an example, we form the problem of discrete optimal control with multi-point boundary conditions $[1,4]$. Let the motion of an object be described by the following linear system of finite-difference equations:

$$
x(i+1)=\psi(i) x(i)+\Gamma(i) u(i) \quad(i=0,1, \ldots, l-1),
$$

with nonseparate boundary conditions

$$
\Phi_{1} x(0)+\Phi_{2} x(s)+\Phi_{3} x(l)=q .
$$

Here $x(l)$ is an $n$-dimensional phase vector, $u(i)$ an $m$-dimensional vector of control influences, $\psi(i), \Gamma(i)(i=0,1, \ldots, l-1)$ matrices of the corresponding dimensions, being a controllability pair $[4,6], \Phi_{1}, \Phi_{2}, \Phi_{3}$ are constant matrices, such that the system (2) satisfies the Kronecker-Capelli condition [3, 4], $0<s<l$.

It is required to find such a control $u(i)$ as minimizes the following quadratic functional:

$$
J=\sum_{i=0}^{l-1}\left(x^{\prime}(i) Q(i) x(i)+u^{\prime}(i) C(i) u(i)\right),
$$

(c) 2016 Aliev. This article is distributed under the terms of the Creative Commons Attribution 4.0 International License (http://creativecommons.org/licenses/by/4.0/), which permits unrestricted use, distribution, and reproduction in any medium, provided you give appropriate credit to the original author(s) and the source, provide a link to the Creative Commons license, and indicate if changes were made. 
under the conditions (1), (2), where $Q(i)=Q^{\prime}(i) \geq 0, C(i)=C^{\prime}(i) \geq 0$ are the periodic matrices of the corresponding dimensions.

Let us illustrate this on the example from [4] in the one-dimensional case. Indeed, in the problem (23)-(25) from [1], let

$$
\begin{aligned}
& n=1, \quad m=1, \quad \psi(0)=\psi(1)=1, \quad \psi(2)=\psi(3)=2, \\
& \Gamma(0)=\Gamma(1)=\Gamma(2)=\Gamma(3)=1, \quad \Phi_{1}=\Phi_{2}=\Phi_{3}=1, \quad q=1, \\
& Q(0)=Q(1)=Q(2)=Q(3)=1, \quad C(0)=C(1)=C(2)=C(3)=1 .
\end{aligned}
$$

Using the algorithm given in [1] we can see that the 'optimal' phase trajectory and control, respectively, have the form

$$
\begin{aligned}
& x(0)=\frac{6}{19}, \quad x(1)=\frac{5}{19}, \quad x(2)=\frac{4}{19}, \quad x(3)=\frac{7}{19}, \quad x(4)=\frac{9}{19}, \\
& u(0)=-\frac{1}{19}, \quad u(1)=-\frac{1}{19}, \quad u(2)=-\frac{1}{19}, \quad u(3)=-\frac{5}{5} .
\end{aligned}
$$

Then it is easy to calculate $[6,7]$ that the 'optimal' value of the functional $(25)$ of [1] will be $J \approx 0.8$.

However, the algorithm as given in $[4,6]$ gives other results, i.e.

$$
\begin{array}{llll}
x(0)=\frac{5}{26}, & x(1)=\frac{1}{26}, & x(2)=\frac{1}{13}, & x(3)=\frac{7}{26}, \quad x(4)=\frac{23}{26}, \\
u(0)=-\frac{2}{13}, & u(1)=\frac{3}{26}, & u(2)=\frac{11}{26}, & u(3)=\frac{9}{26},
\end{array}
$$

and the functional (25) of [1] takes the value

$$
J \approx 0.5 .
$$

Thus, the above solution in [1] is not optimal.

\section{Competing interests}

The author declares that they have no competing interests.

\section{Acknowledgements}

The author thanks the reviewers of the comments and the editors for their instructive remarks.

Received: 8 October 2015 Accepted: 21 March 2016 Published online: 13 May 2016

\section{References}

1. Mutallimov, MM, Zulfugarova, RH, Amirova, Ll: Sweep algorithm for solving optimal control problem with multi-point boundary conditions. Adv. Differ. Equ. 2015, 233 (2015)

2. Abramov, AA: On the transfer of boundary conditions for systems of ordinary linear differential equations (a variant of dispersive method). USSR Comput. Math. Math. Phys. 1(3), 617-622 (1962)

3. Aliev, FA, Larin, VB: On the algorithm for solving discrete periodic Riccati equation. Appl. Comput. Math. 13(1), 46-54 (2014)

4. Aliev, FA: Methods of Solution for the Application Problems of Optimization of the Dynamic Systems. Elm, Baku (1989)

5. Tiwari, S, Kumar, M: An initial value technique solve two-point non-linear singularly perturbed boundary value problems. Appl. Comput. Math. 14(2), 150-157 (2015)

6. Gabasova, OR: On optimal control of linear hybrid systems with terminal constraints. Appl. Comput. Math. 13(2), 194-205 (2014)

7. Rashidinia, J, Khazaei, M, Nikmarvani, H: Spline collocation method for solution of higher order linear boundary value problems. TWMS J. Pure Appl. Math. 6(1), 38-47 (2015) 